\title{
COMUNIDADE DE ARTRÓPODES ASSOCIADA À SERRAPILHEIRA DE CERRADO E MATA DE GALERIA, NA ESTAÇÃO ECOLÓGICA SERRA DAS ARARAS - MATO GROSSO, BRASIL
}

ZARDO, Daniela Cristina. Mestranda do Programa de Pós-Graduação em Recursos Hídricos,

Universidade Federal de Mato Grosso (UFMT). Avenida Fernando Corrêa, Cuiabá-MT. Tel: (65) 36158764. E-mail: daniela_zardo@yahoo.com.br.

CARNEIRO, Ângela Pinheiro. Mestre em Ciências Ambientais, Universidade do Estado de Mato Grosso (Unemat). Rua dos Tucanos, Santa Izabel, Cáceres-MT.

LIMA, Lígia Gonçalves de. Bióloga, Universidade do Estado de Mato Grosso (Unemat). Rua São João, Cavalhada, Cáceres-MT.

SANTOS FILHO, Manoel dos. Professor-doutor da Universidade do Estado de Mato Grosso (Unemat). Rua São João, Cavalhada, Cáceres-MT.

\begin{abstract}
RESUMO
A fauna de artrópodes em serrapilheira destaca-se pela sua importância na ciclagem de nutrientes e degradação da matéria orgânica. Em função disso a fauna de invertebrados tem sido ressaltada como de fundamental importância para os processos que estruturam ecossistemas terrestres. Este estudo tem como objetivos avaliar e comparar a composição em morfoespécies, riqueza e abundância da fauna de artrópodes em serrapilheira de dois ambientes: cerrado e mata de galeria na Estação Ecológica Serra das Araras, Mato Grosso. Para a coleta dos artrópodes foi delimitando um transecto de $120 \mathrm{~m}$ para cada habitat, sendo este divido em seis pontos em cada ambiente. Para todos os pontos de coleta da serrapilheira, demarcou-se uma parcela medindo 1,0 x 1,0 $\mathrm{m}$, totalizando $6,0 \mathrm{~m}^{2}$ por ambiente, onde os artrópodes encontrados foram identificados em nível de ordem agrupando os indivíduos em morfoespécies. Ariqueza total de artrópodes encontrados em áreas de cerrado e mata de galeria foi de 38 morfoespécies, 28 registradas no cerrado e 20, na mata de galeria, com abundância total de 381 indivíduos, com 226 indivíduos coletados no cerrado e 155 indivíduos na mata de galeria. As morfoespécies mais abundantes no cerrado e na mata de galeria foram da ordem Hymenoptera e Isoptera, com 10 e 4 morfoespécies e abundância de 263 e 78 indivíduos, respectivamente. Observa-se que a maior riqueza e abundância de organismos ocorreram no cerrado, isso porque é um ambiente tropical de maior biodiversidade, principalmente no que concerne a sua entomofauna, e essa grande diversidade se refere principalmente à variedade de habitats que o ambiente proporciona, o que confere às espécies abrigo, alimento e sítios de reprodução.
\end{abstract}

Palavras-chave: Cerrado; Biodiversidade; Artrópodos.

\section{Abstract}

The litter arthropod fauna distinguishes itself by its importance in nutrient cycling and organic matter degradation. This invertebrate fauna has been emphasized as crucial for the processes that structure ecosystems. This study aims to evaluate and compare the arthropod fauna composition, richness and abundance in litter of two environments: the savanna and the gallery forest at Serra das Araras Ecological Station, Mato Grosso. To collect the arthropods a $120 \mathrm{~m}$ transects for each habitat was delimited, divided into six points in each environment. For all the litter collection points, we marked a plot measuring $1.0 \times 1.0 \mathrm{~m}$, totalizing $6 \mathrm{~m} 2$ for an environment, where the arthropods found were identified by order level, and the individuals were grouped into morphospecies. The total arthropods richness found in the savanna and in the gallery forest areas was 38 morphospecies, 28 morphospecies were found in the savanna and 20 in the gallery forest, with total abundance of 381 individuals, being 226 individuals collected in the savanna and 155 individuals in the gallery forest. The more abundant 
morphospecies in the savanna and gallery forest belonged to Hymenoptera and Isoptera orders, with 10 and 4 morphospecies and abundance of 263 and 78 individuals respectively. The greatest organism richness and abundance occurred in the savanna, because it is a tropical environment with the greatest biodiversity, especially regarding its insect fauna, and this diversity is primarily concerned with the variety of habitats that the environment provides, which gives the species shelter, food and reproduction sites.

KEYwORDs: Savanna; Biodiversity; Arthropods.

\section{INTRODUÇÃo}

O cerrado brasileiro é reconhecido como a savana mais rica do mundo em biodiversidade, com a presença de diversos ecossistemas. Caracteriza-se por suas diferentes paisagens, tomando por base seus aspectos ecológicos, fisionômicos e, segundo a ordem crescente de sua produção de biomassa, são distinguidas quatro tipos de vegetação: campo limpo, campo sujo, campo cerrado e cerradão (LOPES, 1984; GUARIM NETO $\&$ MORAIS, 2003). Ao longo dos rios de pequeno e médio porte, e córregos dos planaltos do Brasil central, a formação florestal é conhecida como mata de galeria (MORENO \& SCHIAVINI, 2001).

O cerrado caracteriza-se por apresentar variações fisionômico-florísticas consideráveis, desde formas florestais densas até campos puramente herbáceos (COUTINHO,1978), apresentando um teor médio de matéria orgânica e recebendo um incremento anual de resíduos orgânicos provenientes da deposição de folhas durante a estação seca (MARIATH \& SANTOS, 2006). Amata de galeria é caracterizada como floresta tropical sempre verde, sua fisionomia é comumente associada aos solos hidromórficos, com excesso de umidade na maior parte do ano devido ao lençol freático superficial e grande quantidade de material orgânico acumulado, propiciando a decomposição que confere a cor preta característica desses solos (MARIATH \& SANTOS, 2006). Sendo assim, esses dois ambientes apresentam características que favorecem o acúmulo de serrapilheira, constituída de matéria orgânica vegetal e animal que é depositada sobre o solo, sob diferentes estágios de decomposição (BARBOSA \& FARIA, 2006).

A serrapilheira abriga uma enorme diversidade de artrópodes terrestres, integrantes de um grupo que desempenha inúmeras funções ecológicas nos ecossistemas e são importantes para a conservação e manejo de reservas biológicas. A composição e a estrutura das comunidades de artrópodes de serrapilheira são influenciadas por condições ambientais como umidade, tipo de formação vegetal, volume e profundidade de serrapilheira (CORREIA \& OLIVEIRA, 2000).

A fauna de artrópodes em serrapilheira destaca-se pela sua importância na ciclagem de nutrientes e degradação da matéria orgânica, já que esses organismos são os principais responsáveis pela fragmentação da serrapilheira acumulada proveniente da vegetação circundante (SEASTEDT, 1984; MOORE et al., 1991). A comunidade de artrópodes também responde quali e quantitativamente às mudanças ambientais e estas respostas podem afetar o processo de decomposição, alterando, assim, todo o funcionamento de um dado ecossistema, já que seus principais processos, como produtividade primária, serão modificados (RICHARDS, 1974; PRIMAVESI, 1982; SCHOWALTER et al., 1986).

Muitos artrópodes são considerados bons bioindicadores de distúrbio ambiental devido ao fato de responderem rapidamente às mudanças ocorridas no ambiente, apresentarem ampla distribuição geográfica e serem capazes de demonstrar um eficiente gradiente de resposta em função do grau da perturbação (NOSS, 1990; PAOLETTI \& BRESSAN, 1996; BADJI et al. 2004).

Alguns estudos mostram que diferentes grupos de artrópodes respondem diferentemente a alterações nas condições ambientais. Por exemplo, abundantes grupos de Araneae, Formicidae e Coleoptera correlacionamse positivamente com a profundidade da serrapilheira, enquanto o grupo Collembola parece não ser influenciada por esse fator ambiental.

Recentemente, a fauna de invertebrados tem sido 
ressaltada como de fundamental importância para os processos que estruturam ecossistemas terrestres, especialmente nos trópicos (WILSON, 1987), o que explica o crescente número de estudos das comunidades de invertebrados e da utilização desses dados na formulação de estratégias de conservação e diretrizes para o manejo de ecossistemas (SIVESTRE, 2000).

Com base nas informações acima, este estudo apresenta como principal objetivo comparar a composição em morfoespécies, riqueza e abundância da fauna de artrópodes em serrapilheira de dois ambientes: cerrado e mata de galeria na Estação Ecológica Serra das Araras - Eesa, Mato Grosso.

\section{Material e Método}

\section{Local do Estudo}

Este estudo foirealizado na Estação Ecológica Serra das Araras - Eesa, localizada no Estado do Mato Grosso, nos municípios de Porto Estrela, Cáceres e Barra dos Bugres. Aárea é caracterizada por clima do tipo tropical quente e semiúmido, com quatro a cinco meses de seca ao ano. A temperatura média anual é de $24^{\circ} \mathrm{C}$ e a pluviosidade, de 1.400 a $1.500 \mathrm{~mm} /$ anuais. A estimativa porcentual de cada tipo de vegetação em relação à área da unidade resume-se em 50\% de cerrado, $40 \%$ de matas, $5 \%$ de capoeiras, $4 \%$ de campos e cerca de $1 \%$ de várzeas e veredas.

\section{Coleta da fauna}

O estudo foi realizado no fim da estação chuvosa e início da seca de 2007. Para a coleta dos artrópodes de serrapilheira foram selecionados dois ambientes, cerrado e mata de galeria.

Em cada habitat foi delimitado um transecto de 120 $\mathrm{m}$, sendo este divido em seis pontos em cada ambiente. Para evitar o efeito da ação antrópica, as coletas foram realizadas adentrando $15 \mathrm{~m}$ da borda das trilhas já existentes na Eesa.

Para todos os pontos de coleta da serrapilheira, demarcou-se uma parcela medindo 1,0 x 1,0 m (1,0 $\mathrm{m}^{2}$ ), utilizando um quadrado feito de madeira, com um total de $6,0 \mathrm{~m}^{2}$ por ambiente. O material coletado foi levado em sacos plásticos do campo para o laboratório, onde foi realizada triagem manual comauxílio de pinças. Os indivíduos encontrados foram transferidos para sacos plásticos e recipientes de vidro, sendo devidamente etiquetados. Os artrópodes encontrados foram identificados em nível de ordem segundo Brandão \& Cancello (1999).

Foi calculada a porcentagem de abertura de dossel dentro da floresta, onde foi estabelecido um transecto de $120 \mathrm{~m}$, marcado com estacas a cada $20 \mathrm{~m}$. Nesses pontos, a uma distância do solo de 1,60 m, foram tiradas fotos digitais, sempre por uma mesma pessoa. A máquina digital utilizada foi Canon Power Shot A80 (4 megapixels) e a abertura e a velocidade do diafragma foram padronizadas por fotômetro automático.

\section{Análise dos dados}

A porcentagem de abertura de dossel obtida com a câmera digital foi estimada a partir da proporção de pixels brancos na imagem com o auxílio do programa Adobe Photoshop.

Para testar as diferenças entre as variáveis ambientais, bem como entre as abundâncias dos grupos funcionais de artrópodes, foi utilizado o teste de Correlação de Pearson. As médias foram comparadas pelo teste de Tukey-nível de 5\% de probabilidade.

\section{Resultados e Discussão}

A riqueza total de artrópodes encontrados emáreas de cerrado e mata de galeria foi de 38 morfoespécies, com abundância total de 381 indivíduos (Tabela 1). Quando considerada apenas a mata de galeria, a riqueza foi de 20 morfoespécies; o cerrado apresentou riqueza de 28 morfoespécies (Figura 1).

Estudo realizado por Rocha et al. (2010) no município de Anápolis-GO também encontrou maior riqueza no cerrado, com 224 morfoespécies coletadas, sendo 151 no cerrado e 149 na mata de galeria.

O cerrado constitui uma das savanas tropicais de maior biodiversidade, principalmente no que concerne a sua entomofauna (PEREIRA, 2001). Essa grande diversidade de espécies encontradas não se refere somente ao número de espécies, mas tambémà 
variedade de habitats e de paisagens, o que proporciona abrigo e uma ampla distribuição das espécies conforme a preferência por habitats específicos e de acordo com os recursos disponíveis, explicando a maior diversidade nesse ambiente.

Em relação à abundância, o cerrado apresentou 226 espécimes, representando 59,3\% dos indivíduos coletados, e a mata de galeria, 155 espécimes, representando $40,6 \%$ dos indivíduos coletados. As morfoespécies mais abundantes na mata de galeria e no cerrado foram da ordem Hymenoptera, com $117 \mathrm{e}$ 146 indivíduos, respectivamente, e da ordem Isoptera, com 18 e 60 indivíduos, respectivamente (Figura 2).

Nos ecossistemas florestais naturais é encontrada uma abundante comunidade de artrópodes, sendo a macrofauna dominada por insetos sociais (formigas e cupins) e associados à serrapilheira (CORREIA \& OLIVEIRA, 2000).

Estudos realizados em serrapilheira no cerrado por Souza et al. (2007) e Rocha et al. (2010) também registraram a ordem Hymenoptera como a mais abundante nesse ambiente, com $92,41 \%$ e $70,40 \%$ dos indivíduos coletados, respectivamente. Esse resultado já era previsto, uma vez que as formigas (ordem Hymenoptera) são consideradas animais dominantes na maioria dos ecossistemas terrestres (ALONSO \& AGOSTI, 2000). Para as formigas, o volume da serrapilheira está diretamente ligado à quantidade de sítios de nidificação e de alimento disponível; portanto, esta pode ser a causa pela qual a ordem Hymenoptera apresentou maior abundância.

Em relação à maior abundância da ordem Isoptera, Pinheiro et al. (2007) registrou 43 morfoespécies dessa ordem no cerrado em Goiás, o que, segundo o autor, é a riqueza semelhante àquela estimada para o bioma cerrado.

Os cupins (ordem Isoptera) são importantes componentes dafauna do solo, exercendo papel essencial nos processos de decomposição e de ciclagem de nutrientes (AQUIAR et al., 2005). Isso pode confirmar que a composição de espécies dessa ordem se mostrou influenciada pelo tipo fisionômico da vegetação, que, por sua vez, se diferencia principalmente pelo tipo do solo, explicando a maior abundância no ambiente cerrado.
A fauna encontrada associada à serrapilheira depositada no solo é composta, no geral, por espécies que utilizam esse recurso como abrigo e refúgio de predadores. Fatores como luminosidade, umidade e profundidade da serrapilheira influenciam diretamente na riqueza, abundância e distribuição da fauna de artrópodes nesse habitat (CORREIA \& OLIVEIRA, 2000).

As ordens que apresentaram menor abundância na mata de galeria foram Acarina, Ortoptera, Coleoptera e Lumbriculida. E as ordens menos frequentes no cerrado foram Coleoptera, Chilopoda, Diptera, Lepidoptera, Isopoda, Ortoptera e Mesogastrópoda.

Segundo Souza et al. (2007), a presença de alguns grupos taxonômicos, como Diptera e Coleoptera, pode ser considerada atípica em serapilheira, pois esses grupos aparentemente não apresentam nenhuma associação funcional com o subsistema decompositor. Contudo, esses animais utilizam o folhiço como abrigo, sítio de reprodução, ou para forragear.

As morfoespécies que só ocorreram no cerradão foram das ordens Lepidoptera, Mesogastropoda e Diptera, sendo que apenas estas apresentaram formas larvais entre os indivíduos coletados e, por essa razão, possivelmente foram encontradas na serrapilheira de solo. As que só ocorreram na mata de galeria foram da ordem Lumbriculida e Acarineae, que, segundo Storer et al. (2003), é numerosa em solos bons, ricos em humo e umidade abundante, mas raras em ambientes pobres, ácidos, arenosos ou secos como o cerrado. Possivelmente essa ordem tenha sido encontrada na mata de galeria pelas características favoráveis que ela apresenta estando sempre verde, e com excesso de umidade na maior parte do ano (MARIATH \& SANTOS, 2006).

Houve correlação entre a abertura de dossel e volume de serrapilheira nas duas áreas estudadas (Correlação de Pearson $\mathrm{R}=0,302$ ) - quanto maior a abertura do dossel, maior o volume de serrapilheira disponível. Segundo Dias et al. (2004), o volume propiciado pela queda das folhas aumenta a abertura do dossel alterando as condições ambientais dos organismos, pois amplia a luminosidade e a temperatura. 
As variáveis volume da serrapilheira e abertura do dossel não influenciaram significativamente na riqueza $\left(r^{2}=0,040 ; p=0,833\right)$, tampouco na abundância de espécies $\left(r^{2}=0,099 ; p=0,624\right)$. Já estudos realizados por Bieber \& Santos (2004) e Cunha (2004) mostram que o volume e o peso seco de serrapilheira disponível exercem influência na abundância e na riqueza de morfoespécies de artrópodes. Talvez neste estudo não se tenha encontrado essa relação pelo fato de a coleta ter sido realizada na estação seca, em que, segundo Odum \& Barrett (2007), os insetos são mais abundantes na estação chuvosa. Por outro lado, os trabalhos de Bieber \& Santos (2004) e de Cunha (2004) foram desenvolvidos na Amazônia, onde as condições climáticas e ambientais são diferentes, sendo as florestas úmidas mais ricas em serrapilheira.

As características ambientais analisadas nos ecossistemas cerrado e mata de galeria não apresentaram diferença significativa com relação à abertura do dossel, riqueza e abundância, exceto com relação ao volume de serrapilheira, que variou de 5,10 a 11,25 dm³ .

A mata de galeria apresenta altura média arbórea de 20 a 30 m, com sobreposição das copas, oferecendo cobertura de 70 a $95 \%$, enquanto no cerrado o estrato arbóreo apresenta altura média de 8 a 15 metros, com dossel predominantemente contínuo e cobertura de 50 e 90\% (RIBEIRO \& WALTER, 1998).

O cerrado apresentou maior volume de serrapilheira por abrigar árvores geralmente decíduas, que perdem suas folhas durante a estação seca (RAVEM et al., 2001) - o acúmulo ótimo de serrapilheira é necessário para que ocorram queimadas, pois o fogo é parte importante desse ambiente para germinação de sementes e eliminação de árvores invasoras (ODUM \& BARRETT, 2007).

Estudos realizados por Silva et al. (2007) indicam máxima produção de serrapilheira no período de seca. A ocorrência de maior produção de serrapilheira no período seco é influenciada principalmente pelo estresse hídrico, podendo ser uma resposta da vegetação que, com a derrubada de folhas, reduziria a perda de água por transpiração. Já a mata de galeria apresenta folhas sempre verdes, ocorrendo ao longo das margens dos rios e não sofrendo grandes alterações de volume de serrapilheira (ODUM \& BARRETT, 2007).

\section{Conclusão}

Houve correlação entre a abertura de dossel e o volume de serrapilheira nos dois ambientes estudados. Isso porque quanto maior a abertura do dossel, maior o volume de serrapilheira, principalmente durante o período com maior estresse hídrico. A serrapilheira oferece abrigo e alimentação para grande parte da fauna de invertebrados nessas áreas; no entanto, neste estudo o volume da serrapilheira e a abertura do dossel não influenciaram significativamente na riqueza e abundância de espécies.

Apesar de em números totais o cerrado ter tido maior riqueza e abundância, esses valores não foram significativos. Os dois ambientes são bastante complexos quanto à estrutura do habitat, porém, considerando as variáveis umidade e luminosidade, eram esperarados valores maiores na mata de galeria.

Quanto à composição de espécies, apenas quatro não foram comuns, demonstrando certa semelhança na estrutura dos dois habitats. Talvez um estudo em longo prazo possa apresentar resultados diferentes quanto aos ambientes avaliados.

\section{REFERÊNCIAS}

ALONSO, L. E. \& AGOSTI, D., 2000, Biodiversity studies, monitoring, and ants: an overview. In: D. Agosti, J. D. Majer, L. E. Alonso \& T. R. Schultz. Ants: Standard methods for measuring and monitoring biodiversity. Smithsonian Institution Press, Washington, pp. 1-8

AQUIAR, L. M. S.; CAMARGO, A. J. A.; SOUSA, E. S. Fauna de insetos do Cerrado. Agência de informações Embrapa: Bioma cerrado. EMBRAPA, 2005.

BADJI, C.A.; GUEDES, R.N.C.; SILVA, A.A.; ARAUJO, R. A. 2004. Impact of deltamethrin on arthropods in maize under conventional and notillage cultivation. Crop Protection 3: 1031-1039. 
BARBOSA, J. H. C.; FARIA, S. M. Porte de serrapilheira ao solo em estágios sucessionais florestais na reserva biológica de poço das antas, Rio de Janeiro, Brasil. Revista do Jardim Botânico do Rio de Janeiro, v. 57, n. 3, p. 461. 2006.

BRANDÃO, C. R. F.; CANCELLO, E. M. Invertebrados Terrestres. In: JOLY, C. A.; BICUDO, C. E. M. (Orgs). Biodiversidades do estado de São Paulo. Síntese do conhecimento ao final do século XX. São Paulo: Fundação de Amparo à Pesquisa do Estado de São Paulo (FAPESP), 1999, v. 5, XVIII 279p.

BIEBER, A. G. D; SANTOS, B. A. Comunidade de artrópodes associada à serrapilheira acumulada sobre folhas de duas espécies de palmeira em uma floresta de terra-firme na Amazônia central. 2004. Curso de campo: Ecologia da Floresta Amazônica. Disponível em: http://www.inpa.gov.br/ pdbff/download/efa/livro/ 2004/PDFs/pl1g1.pdf>. Acesso em: 18 de Nov. 2007.

COUTINHO, L.M. 1978. O conceito de Cerrado. Revista Brasileira de Botânica 7:17-23.

CORREIA, M. E. F; OLIVEIRA, L. C. M. Fauna de Solo: Aspectos Gerais e Metodológicos. Seropédica: EmbrapaAgrobiologia, 2000.p. 46. Disponívelem: <http://www.cnpab.embrapa.br/servicos/download/ doc112.pdf>. Acesso em: 20 jul. 2007.

CUNHA, N. L. Artropódos associados à serrapilheira suspensa acumulada em folhas de duas palmeiras, Amazônia Central. 2004. Curso de campo: Ecologia da Floresta Amazônica.

Disponível em: <http://www.inpa.gov.br/ pdbff/ download/efa/livro/2004/PDFs /41_final/ nicolay.pdf> . Acesso em: 18 nov. 2007.

DIAS, S. C.; SILVA, W. R.; MARTINS E. G.; OSSES, F.; BALDISSERA, R. Grupos funcionais de artrópodes de serrapilheira de dois ambientes em uma área de extração de madeira na Amazônia Central. 2004. Curso de campo: Ecologia da Floresta Amazônica. Disponível em: <http:// www.inpa.gov.br/ pdbff/download/efa/livro/2004/ PDFs/pl1g6.pdf>. Acessado em: 18 nov. 2007.

GUARIM NETO, G.; MORAIS, R. G. Recursos medicinais de espécies do Cerrado de Mato Grosso: um estudo bibliográfico. Acta Botânica Brasileira, v. 17, n. 4, p. 561-584. 2003.

\section{LOPES, A. S. Solos sob "Cerrado":}

Características propriedades e manejo. ed. 2, Piracicaba: Potafos, 1984.

MARIATH, A. E. J.; SANTOS, P. R. (org.). Os avanços da botânica no inicio do século XXI: morfologia, fisiologia, taxonomia, ecologia e genética. Conferências Plenárias e Simpósios do $57^{\circ}$ Congresso Nacional de Botânica - Porto Alegre: Sociedade Botânica do Brasil. 2006. 752p.

MOORE, J. C., HUNT, H. W. ; ELLIOTTI, E. T.. Interactios between soil organisms and herbivores. In: P. Barbosa, V. Kirschik and C. Jones (eds.) Multitrophic-level interactions among microorganisms, plants and insects. New York: John Wiley, 385p.

MORENO, M. I. C.; SCHIAVINI, I. Relação entre vegetação e solo em um gradiente florestal na Estação Ecologia do Panga, Uberlândia (MG). Revista Brasileira de Botânica, v. 24, n. 4, p. 537-54. 2001.

NOSS, R.F. 1990. Indicators for monitoring biodiversity: a hierarchiacal approach. Conserv. Biol. 4: 355-364.

ODUM, E. P.; BARRETT, G. W. Fundamentos de Ecologia. 5. ed. São Paulo: Thomson Learning, 2007. PAOLETTI, M.G. \& M. BRESSAN. 1996. Soil 
invertebrates as bioindicators of human disturbance. Crit. Rev. Plant Sci. 15: 21-62.

PEREIRA, H.S. 2001. Cambio em el uso de la Tierra, Tema: Conservação de Áreas Florestais, Brasil. Proyecto Infomacion y Analisis para el Manejo Forestal Sostenible: Integrando Esfuerzos Nacionales e Internacionales en 13 Paises Tropicales en America Latina. Santiago, Chile. 18p.

PINHEIRO, D. G.; BRANDÃO, D.; SANTOS T. \& BARBOSA REZENDE, P. Padrões de Diversidade de Isoptera em áreas de cerrado no Município de Niquelândia. CONGRESSO DE ECOLOGIA DO BRASIL, 8. Anais... Goiás, 23 a 28 de Setembro de 2007, Caxambu-MG.

PRIMAVESI, A. 1982. O manejo ecológico do solo. Livraria Nobel, SA, 541p.

RAVEN, P. H.; EVERT, R. F.; EICHHORN, S. E. Biologia Vegetal. 6. ed. Rio de Janeiro: Guanabara Koogan, 2001.

RIBEIRO, J. F; WALTER, B. M. T. Fitofisionomias do bioma cerrado. In: SANO, S. M.; ALMEIDA, S. P. (eds). Cerrado: ambiente e flora. EMBRAPACPAC, Planaltina, p.89-166. 1998.

RICHARDS, B.N. 1974. Introduction to the soil ecosystem. Longman Group Ltd. New York, $266 \mathrm{p}$.

ROCHA, G. O.; NETTO, M. C. B., LOZI, L. R. P.. Diversidade, riqueza e abundância da entomofauna edáfica em área de cerrado do Brasil Central. Universidade Estadual de Goiás, Anápolis, GO. Disponível em: http://www.sebecologia.org.br/viiceb/resumos/1036a.pdf. Acessado dia 07 de fevereiro de 2010.
SCHOWALTER, T.D., HARGROVE, W.W. \& CROSSLEY, D.A.. JR. 1986. Herbivory in forested ecosystems. Annu. Rev. Entomol. 31: 177-196.

SEASTED, T. R. 1984. The role of microarthropods $\mathrm{n}$ decomposition and mieralization processes. Annu. Rev. Entomol, v.29, p.25-46.

SILVA, C. J.; SANCHES, L.; BLEICH, M. E.; LOBO, F.A.; NOGUEIRA, J. S. Produção de serrapilheira no Cerrado e Floresta de Transição Amazônia-Cerrado do Centro-Oeste Brasileiro. Acta Amazônica, v. 37, n. 4. 2007.

SILVESTRE, R. 2000. Estrutura de comunidades de formigas do Cerrado. Tese de Doutorado. Programa de Pós-graduação em entomologia. Faculdade de Filosofia, Ciências e Letras de Ribeirão Preto, Departamento de Biologia. Universidade de São Paulo. 204p.

SOUZA, C. M. de; SOUZA, V. B. de; AMÂNCIO, S.; SALOMÃO, C.; PEDROSO, E. T.; AUGUSTO, S. C. Artrópodes associados à serapilheira e troncos de árvores suberosas em uma área de cerradão em Uberlândia, MG. CONGRESSO DE ECOLOGIA, 8. Anais. 23 a 28 de Setembro de 2007.

STORER, T. I.; USINGER, R. L.; STEBBINS, R. C.; NYBAKKEN, W. J. Zoologia Geral. 6 ed. São Paulo: Campanha Editora Nacional, 2003.

VASCONCELOS, H. L. Effects of litter collection by understory palms on the associated macroinvertebrates fauna in Central Amazonia. Pedobiologia, v. 34, p. 157-160. 1984.

WILSON, E.O. 1987. The Little Things that Run the World: The Importance and Conservation of invertebrates. Conservation Biology, 1: 344-346. 
Tabela 1 - Tabela com o número de ordens e morfoespécies encontradas nos ambientes estudados (mata de galeria e cerrado), e os valores de riqueza e abundância, na Estação Ecológica Serra das Araras-MT.

\begin{tabular}{|c|c|c|c|c|c|}
\hline Classe & Ordem & Morfo-espécie & Cerradão & $\begin{array}{l}\text { Mata de } \\
\text { Gal eria }\end{array}$ & Total \\
\hline \multirow[t]{22}{*}{ Insecta } & Hy menoptera & Hymenoptera sp1 & 14 & 31 & 45 \\
\hline & & Hymenoptera sp2 & 1 & 72 & 73 \\
\hline & & Hymenoptera sp3 & 19 & 8 & 27 \\
\hline & & Hymenoptera sp4 & 0 & 2 & 2 \\
\hline & & Hymenoptera sp5 & 0 & 2 & 2 \\
\hline & & Hymenoptera sp6 & 0 & 2 & 2 \\
\hline & & Hymenoptera sp7 & 2 & 0 & 2 \\
\hline & & Hymenoptera sp8 & 1 & 0 & 1 \\
\hline & & Hymenoptera sp9 & 89 & 0 & 89 \\
\hline & & Hymenoptera sp10 & 20 & 0 & 20 \\
\hline & Orthoptera & Orthoptera sp1 & 2 & 2 & 4 \\
\hline & & Orthoptera sp2 & 1 & 2 & 3 \\
\hline & Diptera & Diptera sp1 & 1 & 0 & 1 \\
\hline & & Diptera sp2 & 1 & 0 & 1 \\
\hline & Lepidoptera & Lepidoptera sp1 & 1 & 0 & 1 \\
\hline & Isoptera & Isoptera sp 1 & 15 & 18 & 33 \\
\hline & & Isoptera sp2 & 19 & 0 & 19 \\
\hline & & Isoptera sp3 & 7 & 0 & 7 \\
\hline & & Isoptera sp4 & 19 & 0 & 19 \\
\hline & Coleoptera & Coleoptera sp1 & 1 & 1 & 2 \\
\hline & & Coleoptera sp2 & 0 & 1 & 1 \\
\hline & & Coleoptera sp3 & 0 & 1 & 1 \\
\hline Crustacea & Isopoda & Isopoda sp1 & 1 & 0 & 1 \\
\hline \multirow[t]{8}{*}{ Arachida } & Araneae & Araneae sp1 & 1 & 2 & 3 \\
\hline & & Araneae sp2 & 2 & 2 & 4 \\
\hline & & Araneae sp 3 & 0 & 3 & 3 \\
\hline & & Araneae sp4 & 0 & 2 & 2 \\
\hline & & Araneae sp5 & 1 & 1 & 2 \\
\hline & & Araneae sp6 & 2 & 0 & 2 \\
\hline & & Araneae sp7 & 1 & 0 & 1 \\
\hline & Acarina & Acarina sp1 & 0 & 1 & 1 \\
\hline \multirow[t]{2}{*}{ Chilopoda } & Chilopoda & Chilopoda sp 1 & 1 & 0 & 1 \\
\hline & & Chilopoda sp2 & 1 & 0 & 1 \\
\hline \multirow[t]{3}{*}{ Gastropoda } & Mesogas trópoda & $\begin{array}{l}\text { Mes ogas trópoda } \\
\text { sp1 }\end{array}$ & 1 & 0 & 1 \\
\hline & & $\begin{array}{l}\text { Mes ogas trópoda } \\
\text { sp2 }\end{array}$ & 1 & 0 & 1 \\
\hline & & $\begin{array}{l}\text { Mes ogas trópoda } \\
\text { sp3 }\end{array}$ & 1 & 0 & 1 \\
\hline \multirow[t]{2}{*}{ Oligochaeta } & Lumbriculida & Lumbriculida sp1 & 0 & 1 & 1 \\
\hline & & Lumbriculida sp2 & 0 & 1 & 1 \\
\hline Riqueza total & & & 28 & 20 & 38 \\
\hline Abundância total & & & 226 & 155 & 381 \\
\hline
\end{tabular}

Fonte: Dados de pesquisa. 
Comunidade de artrópodes...

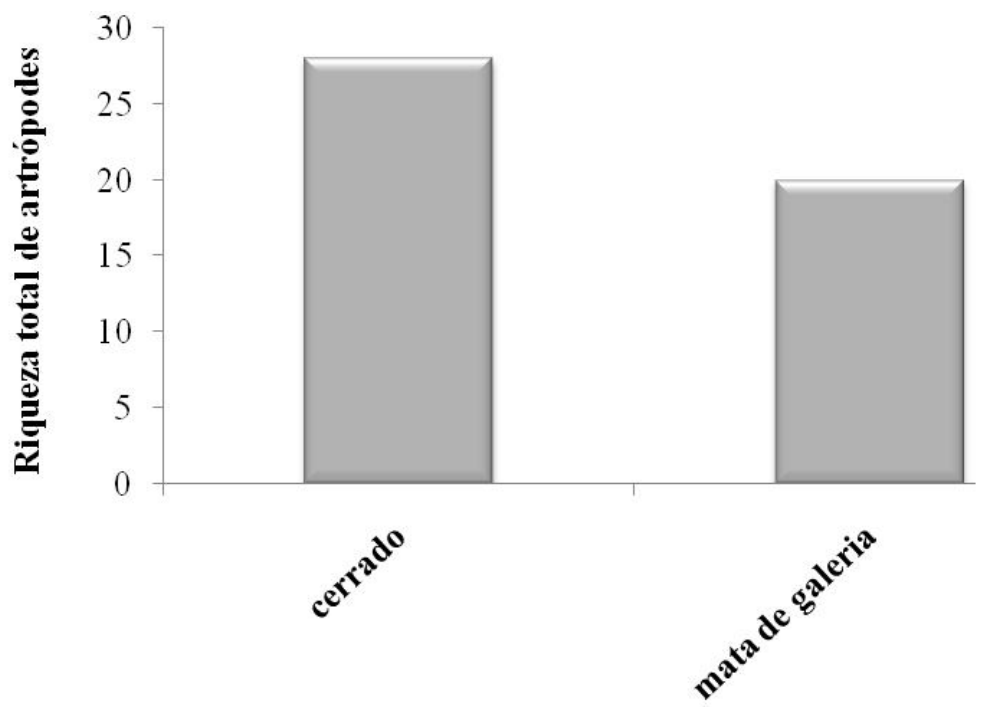

Figura 1 - Riqueza total de artrópodes coletados nos ambientes de cerrado e mata de galeria na Estação Ecológica Serra das Araras-MT.

Fonte: Dados de pesquisa.

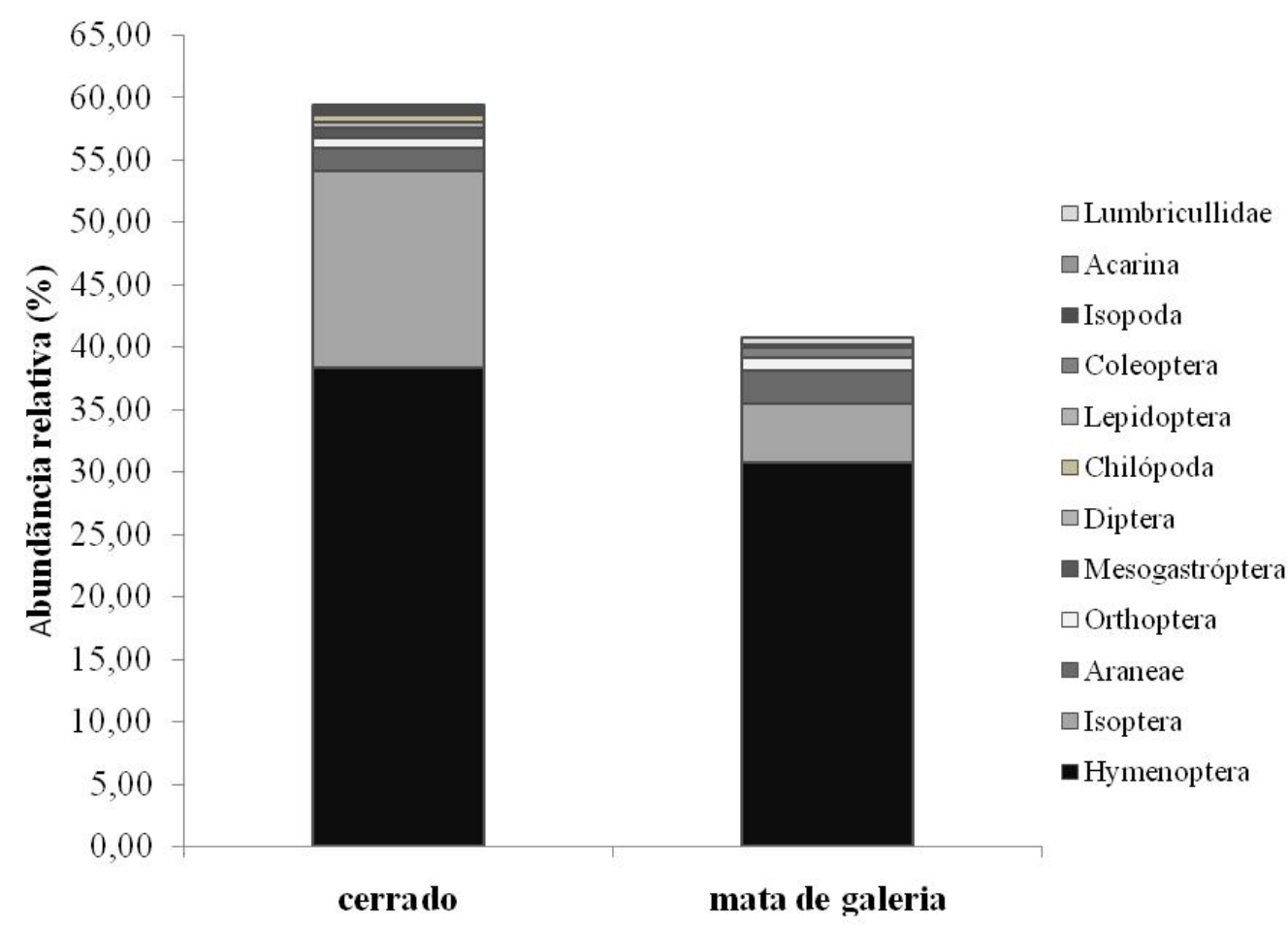

Figura 2 - Abundância relativa das ordens coletadas nos ambientes de cerrado e mata de galeria na Estação Ecológica Serra das Araras-MT.

Fonte: Dados de pesquisa.

RECEBIDO EM 30/11/2010

ACEITO EM 15/12/2010 